TAIWANESE JOURNAL OF MATHEMATICS

Vol. 7, No. 3, pp. 507-512, September 2003

This paper is available online at http://www.math.nthu.edu.tw/tjm/

\title{
A DOMINATED CONVERGENCE THEOREM IN THE K-H INTEGRAL
}

\author{
Jitan Lu and Peng-Yee Lee
}

\begin{abstract}
In this paper, we give a nonabsolute dominated convergence theorem for the K-H integral on the real line. Furthermore, as the converse part, we also give a corresponding Riesz type definition of the K-H integral.
\end{abstract}

\section{INTRODUCTION}

The dominated convergence theorem (see for example [6, Theorem 9.20]) plays an important role in the Lebesgue integral. A dominated convergence theorem for the K-H integral is also known (see [2, Theorem 8.12]). But unfortunately it was proved to be absolute. Although some nonabsolute dominated convergence theorems were given later (see [3, Theorem 5.5.5] and [5, Theorem 3]), we have never seen that any Riesz type definition of the $\mathrm{K}-\mathrm{H}$ integral corresponding to some dominated convergence theorem was given. In this paper, we give another dominated convergence theorem, which is also truly nonabsolute. More than that, as the converse part, we give a corresponding Riesz type definition of the K-H integral.

\section{Preliminaries}

Let $E$ be a closed bounded interval on the real line, if necessary, sometime we write it as $[a, b]$, where $a$ and $b$ are the two end points of $E$. We shall call an subinterval $I$ of $E$ with one of its end points $x,(x, I)$ a point-interval pair. A partial division $D$ of $E$ is a finite collection of point-interval pairs $(x, I)$ with the intervals non-overlapping, and their union forming a subset of $E$. We shall write

Received Jane 23, 2002; revised Auguest 4, 2002.

Communicated by B.-L. Lin.

2000 Mathematics Subject Classification: 26A39.

Key words and phrases: Dominated convergence theorem, the K-H integral, Major function, Minor function, Riesz type definition of the K-H integral. 
$D=\{(x, I)\}$. If a partial division $D$ is such that the union of the intervals in $D$ is $E$, we call $D$ a division of $E$.

Let $\delta: E \rightarrow(0,1)$ be a function on $E$ and we call it a gauge of $E$. A partial division $D=\{(x, I)\}$ is said to be $\delta$-fine if, for each point-interval pair $(x, I)$, we have $I \subset[x-\delta(x), x+\delta(x)]$. We recall that a real-valued function $f$ defined on an interval $E$ is said to be Kurzweil-Henstock $(\mathrm{K}-\mathrm{H})$ integrable if there exists a real number $A$, for every $\varepsilon>0$, there is a gauge $\delta$ of $E$, such that for any $\delta$-fine division $D=\{(x, I)\}$ of $E$, we always have

$$
\left|(D) \sum f(x)\right| I|-A|<\varepsilon,
$$

where $|I|$ denotes the length of the interval $I$ and $(D) \sum$ denotes the sum over all point-interval pairs $(x, I)$ in $D$. It is known that if $f$ is $\mathrm{K}-\mathrm{H}$ integrable on an interval $E$, so is it on any subinterval $I$ of $E$. Then we obtain an interval function $F$ defined on the family of all the subintervals of $E$. We call $F$ the primitive of the $\mathrm{K}-\mathrm{H}$ integrable function $f$. It is also known that $F$ is finitely additive in the sense of that if $I=\cup_{i=1}^{n} I_{i}$ and $I_{i}$ are nonoverlapping intervals then

$$
F(I)=\sum_{i=1}^{n} F\left(I_{i}\right) .
$$

\section{A Dominated Convergence Theorem}

Let $E$ be an interval of the real line, and

$$
\Lambda(E)=\{(x, I):(x, I) \text { is a point-interval pair of } E\},
$$

Let $H$ be a function defined on $\Lambda(E)$ and $X$ a subset of $E$. We say $H$ is $B V^{*}(X)$ if

$$
\sup (D) \sum|H(x, I)|<+\infty .
$$

where the supremum is taken over all partial divisions $D=\{(x, I)\}$ of $E$ with $x \in$ $X$. We say $H$ is $B V G^{*}$ on $E$ if there exists a sequence of $\left\{X_{i}\right\}$ with $\cup_{i=1}^{\infty} X_{i}=E$ such that $H$ is $B V^{*}\left(X_{i}\right)$ for any $i$.

Definition 3.1. Let $F, H$ and $G$ be functions on $\Lambda(E)$. $H$ is said to be a major function of $F$ on $E$ if $H$ is $B V G^{*}$ on $E$ and

$$
H(x, I) \geq F(x, I)
$$

for every $(x, I) \in \Lambda(E) ; G$ is said to be a minor function of $F$ on $E$ if $G$ is $B V G^{*}$ on $E$ and 


$$
F(x, I) \geq G(x, I)
$$

for every $(x, I) \in \Lambda(E)$.

Based on the concepts given above, we have the following dominated convergence theorem.

Ttheorem 3.1. If the following conditions are satisfied:

(1) $f_{n}(x) \rightarrow f(x)$ almost everywhere in $E$ as $n \rightarrow \infty$ where each $f_{n}$ is $K$-H integrable on $E$;

(2) The primitive $F_{n}$ of $f_{n}, n=1,2, \ldots$, have at lease one common major function $H$ and at least one common minor function $G$ on $E$;

(3) $F_{n}$ converge uniformly to a limit function $F$ on $E$, then $f$ is K-H integrable to $F(E)$ on $E$.

We note that the primitive $F$ of a K-H integrable function on $E$ can be treated as a special function defined on $\Lambda(E)$. So the major and minor functions in Theorem 3.1 are meaningful.

Proof of Theorem 3.1. Follow the proof of [2, Theorem 8.12] using standard category argument.

\section{A Riesz-Type Definition of the K-H Integral}

In this section, we will show that the converse part of Theorem 3.1 is also true, which provides a Riesz type definition of the K-H integral. Furthermore, it also shows that Theorem 3.1 is a nonabsolute dominated convergence theorem.

Let $f_{n}$ be a sequence of $\mathrm{K}-\mathrm{H}$ integrable functions on $E$ with the primitive $F_{n}$. We say $\left\{f_{n}\right\}$ is dominated convergent to a function $f$ if all the conditions in Theorem 3.1 hold. Then we have the following result.

Theorem 4.1. Let $f$ be a K-H integrable function on $E$. Then there exists a sequence of Lebesgue integrable functions $\left\{f_{n}\right\}$ which is dominated convergent to $f$.

Proof. Let $F$ be the primitive of $f$ on $E=[a, b]$. Then by [2, Lemma 6.17] $F$ is $V B G^{*}$ on $E$. Hence $E=\cup_{n=1}^{\infty} X_{n}$ and $F$ is $V B^{*}\left(X_{n}\right)$ for each $n$. We assume that $X_{n}$ is closed by [2, Lemma 6.16] and moreover $X_{n} \subset X_{n+1}$ for any $n$.

Define $F_{n}(x)=F(x)$ when $x \in X_{n}, F_{n}(a)=F(a), F_{n}(b)=F(b)$ and

$$
F_{n}(x)=F\left(a_{k}\right)+\frac{F\left(b_{k}\right)-F\left(a_{k}\right)}{b_{k}-a_{k}}\left(x-a_{k}\right) \text { when } x \in\left(a_{k}, b_{k}\right)
$$


where $(a, b)-X_{n}=\cup_{k=1}^{\infty}\left(a_{k}, b_{k}\right)$.

We can prove that $F_{n}$ is absolutely continuous on $E$ (see the proof of [3, Theorem 5.3.13]). So by [2, Theorem 5.5] the derivative $f_{n}(x)=F_{n}^{\prime}(x)$ almost everywhere in $[a, b]$ is absolutely $\mathrm{K}-\mathrm{H}$ integrable, that is Lebesgue integrable on $[a, b]$ with the primitive $F_{n}$. Moreover, it is obvious that $F_{n}$ converges uniformly to $F$ and $f_{n}$ converges to $f$ almost everywhere.

Now we define two functions $H$ and $G$ on $\Lambda(E)$ as follows:

$$
H(x, I)=\sup \left\{F_{n}(I)\right\}
$$

and

$$
G(x, I)=\inf \left\{F_{n}(I)\right\}
$$

for any $(x, I) \in \Lambda(E)$, here the supremum and infimum taking over all $n$. From the fact that $F_{n}$ converges uniformly to $F$, we know that the supremum and infimum exist, and it is obvious that for any $(x, I) \in \Lambda(E)$,

$$
H(x, I) \geq F_{n}(I) \geq G(x, I)
$$

for all $n$.

Now we fix $X_{i}$. We shall prove that both $H$ and $G$ are $V B^{*}\left(X_{i}\right)$.

First we can check that $F_{n}$ is $V B^{*}$ on $E$. For any $j=1,2, \ldots, i-1$, there exists $M_{j}>0$, such that for any partial division $D=\{(x, I)\}$ of $E$, we have

$$
\text { (D) } \sum \omega\left(F_{j} ; I\right) \quad M_{j}
$$

where $\omega\left(F_{j} ; I\right)$ denotes the oscillation of $F$ over $I$, that is,

$$
\omega\left(F_{j} ; I\right)=\sup \left\{\left|F\left(I^{\prime}\right)\right|: I^{\prime} \subset I\right\} .
$$

We see that for any $n \geq i, F_{n}(x)=f(x)$ when $x \in X_{i}$ and that

$$
\sum_{k=1}^{\infty} \omega\left(F_{n} ;\left[a_{k}, b_{k}\right]\right) \quad \sum_{k=1}^{\infty} \omega\left(F ;\left[a_{k}, b_{k}\right]\right)<M^{\prime}
$$

where $(a, b)-X_{i}=\cup_{k=1}^{\infty}\left(a_{k}, b_{k}\right), M^{\prime}$ is a positive number. The existence of $M^{\prime}$ comes from the fact that $F$ is $V B^{*}\left(X_{i}\right)$ and continuous on $E$ (see [3, Lemma 5.3.8]). Certainly we may assume that $M^{\prime}$ is bigger than $M_{n}$ for any $n=1,2, \ldots, i-1$.

Since $F$ is $V B^{*}\left(X_{i}\right)$,then there exist a positive number $M^{\prime \prime}>0$ such that for any partial division $D^{\prime}=\{(x, I)\}$ of $E$ with $x \in X_{i}$, we have

$$
\left(D^{\prime}\right) \sum|F(I)|<M^{\prime \prime}
$$


Let $D=\left\{(x, I): x \in X_{i}\right\}$ be any partial division of $E$. Now we rearrange it as follows.

For any element $(x, I) \in D,(1)$ if both the two ends of $I$ belong to $X_{i}$, leave it alone; (2) if another end point of $I$ does not belong to $X_{i}$, then divide $I$ into two parts $I_{1}$ and $I_{2}$, such that both of the two end points of $I_{1}$ belong to $X_{i}$ and $I_{2}$ is included in some $\left[a_{k}, b_{k}\right]$. All the elements we get in the above two cases form a new partial division $D^{\prime}$ of $E$. We divide $D^{\prime}$ into two parts:

$$
D_{1}=\left\{\left(x^{\prime}, I^{\prime}\right) \in D^{\prime}: I^{\prime} \text { is included in some }\left[a_{k}, b_{k}\right]\right\}
$$

and $D_{2}$ the rest. For any $k$, it is obvious that

$$
\sum_{\substack{\left(x^{\prime}, I^{\prime}\right) \in D_{1} \\ I \subset\left(a_{k}, b_{k}\right)}}\left|F_{n}\left(I^{\prime}\right)\right| \quad 2 \omega\left(F_{n} ;\left[a_{k}, b_{k}\right]\right) \quad 2 \omega\left(F ;\left[a_{k}, b_{k}\right]\right) .
$$

So

$$
\begin{aligned}
& \sum_{\substack{\left(x^{\prime}, I^{\prime}\right) \in D_{1} \\
I^{\prime} \subset\left[a_{k}, b_{k}\right]}}\left|H\left(x^{\prime}, I^{\prime}\right)\right| \\
& \max \left\{2 \omega\left(F ;\left[a_{k}, b_{k}\right]\right), 2 \omega\left(F_{j} ;\left[a_{k}, b_{k}\right]\right): j=1,2, \ldots i-1\right\} .
\end{aligned}
$$

Combining (4.1) and (4.2) and (4.4), we have that

$$
\begin{aligned}
& \left(D_{1}\right) \sum\left|H\left(x^{\prime}, I^{\prime}\right)\right| \\
& 2 \max \left\{\sum_{k=1}^{\infty} \omega\left(F ;\left[a_{k}, b_{k}\right]\right), \sum_{k=1}^{\infty} \omega\left(F_{j} ;\left[a_{k}, b_{k}\right]\right): j=1, \ldots, i-1\right\} \\
< & 2 M^{\prime} .
\end{aligned}
$$

For any interval $I^{\prime}$ in $D_{2}$, it is obvious that

$$
\left|F_{n}\left(I^{\prime}\right)\right|=\left|F\left(I^{\prime}\right)\right|
$$

for any $n \geq i$. Moreover we notice that $D_{2}$ is a partial division of $E$ with $x^{\prime} \in X_{i}$. So (4.3) holds for $D_{2}$. Thus

$$
\left(D_{2}\right) \sum\left|F\left(I^{\prime}\right)\right|<M^{\prime \prime}
$$

Combining (4.1), (4.3), (4.6) and (4.7), we have

$$
\begin{aligned}
& \left(D_{2}\right) \sum\left|H\left(x^{\prime}, I^{\prime}\right)\right| \\
& \left(D_{2}\right) \sum \max \left\{\left|F\left(I^{\prime}\right)\right|,\left|F_{j}\left(I^{\prime}\right)\right|: j=1,2, \ldots, i-1\right\} \\
& \left(D_{2}\right) \sum\left|F\left(I^{\prime}\right)\right|+\left(D_{2}\right) \sum \sum_{j=1}^{i-1}\left|F_{j}\left(I^{\prime}\right)\right|
\end{aligned}
$$




$$
<M^{\prime \prime}+\sum_{j=1}^{i-1} M_{j} .
$$

Now let $M=\max \left\{2 M^{\prime}, M^{\prime \prime}+\sum_{j=1}^{i-1} M_{j}\right\}$. Then for any partial division $D=$ $\left\{(x, I): x \in X_{i}\right\}$ of $E$, combining (4.5) and (4.8), we have

$$
\begin{aligned}
& (D) \sum\left|H\left(x^{\prime} I^{\prime}\right)\right| \\
& \left(D_{1}\right) \sum\left|H\left(x^{\prime} I^{\prime}\right)\right|+\left(D_{2}\right) \sum\left|H\left(x^{\prime} I^{\prime}\right)\right|<M .
\end{aligned}
$$

This means that $H$ is $V B^{*}\left(X_{i}\right)$ for any $i$ and then is $V B G^{*}$ on $E$. Similarly we can prove that $G$ is also $V B G^{*}$ on $E$. Up to now, we have proved that $H$ and $G$ are the common major and minor functions of $F_{n}$ and then $f_{n}$ is a dominated Lebesgue integrable sequence and $f_{n}$ converges to $f$.

\section{REFERENCES}

1. R. Henstock, Lectures on the Theory of Integration, World Scientific, Singapore, 1988.

2. P. Y. Lee, Lanzhou Lectures on Henstock Integral, World Scientific, Singapore, 1989.

3. P. Y. Lee and R. Vyborny, The Integral: An Easy Approach after Kurzweil and Henstock, Cambridge, 2000.

4. Jitan Lu and P. Y. Lee, The primitives of Henstock integrable functions in Euclidean space, Bull. London Math. Soc. 31 (1999), 173-180.

5. Shipan Lu and Kecheng Liao, On generalized dominated convegence, Real Analysis Exchange, 16 (1990-1991), 74-78.

6. J. Yeh, Lectures on Real Analysis, World Scientific, Singapore, 2000.

Jitan $\mathrm{Lu}$

Block 430, \#07-362, Clementi Avenue 3,

Singapore 120430

E-mail: lujitan@hotmail.com

Peng-Yee Lee

Mathematics and Mathematics Education

National Institute of Education

1 Nanyang Walk

Nanyang Technological University

Singapore 637616

E-mail: pylee@nie.edu.sg 